\title{
A Methodology for Extrinsically Evaluating Information Extraction Performance
}

\author{
Michael Crystal, Alex Baron, Katherine Godfrey, Linnea Micciulla, Yvette Tenney, and \\ Ralph Weischedel \\ BBN Technologies \\ 10 Moulton St. \\ Cambridge, MA 02138-1119 \\ mcrystal@bbn.com
}

\begin{abstract}
This paper reports a preliminary study addressing two challenges in measuring the effectiveness of information extraction (IE) technology:

- Developing a methodology for extrinsic evaluation of IE; and,

- Estimating the impact of improving IE technology on the ability to perform an application task.

The methodology described can be employed for further controlled experiments regarding information extraction.
\end{abstract}

\section{Introduction}

Intrinsic evaluations of information extraction (IE) have a history dating back to the Third Message Understanding Conference ${ }^{1}$ (MUC-3) and continuing today in the Automatic Content Extraction (ACE) evaluations. ${ }^{2}$ Extrinsic evaluations of IE, measuring the utility of IE in a task, are lacking and needed (Jones, 2005).

In this paper, we investigate an extrinsic evaluation of IE where the task is question answering (QA) given extracted information. In addition, we propose a novel method for exploring hypothetical performance questions, e.g., if IE accuracy were $\mathrm{x} \%$ closer to human accuracy, how would speed and accuracy in a task, e.g., QA, improve?

\footnotetext{
${ }^{1}$ For more information on the MUC conferences, see http://www.itl.nist.gov/iad/894.02/related_projects/muc/.

${ }^{2}$ For an overview of ACE evaluations see

http://www.itl.nist.gov/iad/894.01/tests/ace/.
}

We plot QA accuracy and time-to-complete given eight extracted data accuracy levels ranging from the output of SERIF, BBN's state-ofthe-art IE system, to manually extracted data.

\section{Methodology}

Figure 1 gives an overview of the methodology. The left portion of the figure shows source documents provided both to a system and a human to produce two extraction databases, one corresponding to SERIF's automated performance and one corresponding to doubleannotated, human accuracy. By merging portions of those two sources in varying degrees ("blends"), one can derive several extracted databases ranging from machine quality, through varying percentages of improved performance, up to human accuracy. This method of blending databases provides a means of answering hypothetical questions, i.e., what if the state-of-theart were $\mathrm{x} \%$ closer to human accuracy, with a single set of answer keys.

A person using a given extraction database performs a task, in our case, QA. The measures of effectiveness in our study were time to complete the task and percent of questions answered correctly. An extrinsic measure of the value of improved IE technology performance is realized by rotating users through different extraction databases and questions sets.

In our preliminary study, databases of fully automated IE and manual annotation (the gold standard) were populated with entities, relationships, and co-reference links from 946 documents. The two initial databases representing machine extraction and human extraction respectively were then blended to produce a continuum of database qualities from machine to 


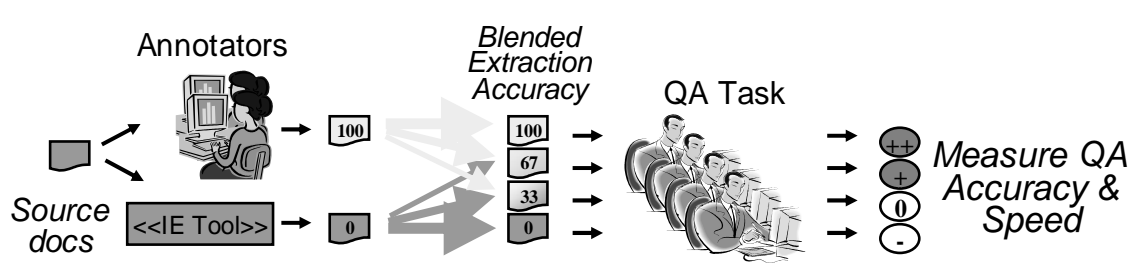

Figure 1: Study Overview

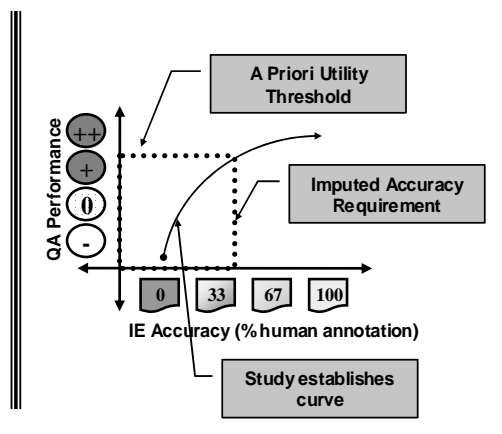

SERIF, BBN's automatic IE system based on its predecessor, SIFT (Miller, 2000), was run on the 946 ACE documents to create the machine (DBquality 0) database. SERIF is a statistically trained software system that automatically performs entity, co-reference, and relationship information extraction.

Intermediate IE performance was simulated by blending the human and automatically generated databases in various degrees using an interpolation algorithm developed specifically for this study. To create a blended database, DBquality $n$, all of the entities, relationships, and co-reference links common to the human and automatically generated databases are copied into a new one. Then, $n \%$ of the entity mentions in the human database (100), but not in the automatic IE system output (0), are copied; and, $(100-n) \%$ of the entity mentions in the automatically generated database, but not in the human database, are copied. Next, the relationships for which both of the constituent entity mentions have been copied are also copied to the blended database. Finally, co-reference links and entities for the already copied entity mentions are copied into the blended database.

For the first experiment, two intermediate extraction databases were created: DB-qualities 33 and 67. For the second experiment, two additional databases were created: 16.5 and 50. The first intermediate databases were both created using the 0 and 100 databases as seeds. The 16.5 database was created by mixing the 0 and the 33 databases in a $50 \%$ blend. The 50 database was created by doing the same with the 33 and 67 databases. For Experiment 3, 41 and 58 databases were created by mixing the 33 and 50, and 50 and 67 databases respectively.

\footnotetext{
${ }^{3}$ The 2004 ACE evaluation plan, available at http://www.nist.gov/speech/tests/ace/ace04/doc/ace04-evalplanv7.pdf, contains a full description of the scoring metric used in the evaluation. Entity type weights were 1 and the level weights were $\mathrm{NAM}=1.0, \mathrm{NOM}=0.5$, and $\mathrm{PRO}=0.1$.
} 


\begin{tabular}{|c|c|c|c|c|c|c|c|c|c|c|c|c|c|c|c|c|}
\hline & \multicolumn{16}{|c|}{ DB Blend } \\
\hline & \multicolumn{2}{|c|}{$\begin{array}{c}0 \\
\text { (Machine) }\end{array}$} & \multicolumn{2}{|c|}{16.5} & \multicolumn{2}{|c|}{33} & \multicolumn{2}{|c|}{41} & \multicolumn{2}{|c|}{50} & \multicolumn{2}{|c|}{58} & \multicolumn{2}{|c|}{67} & \multicolumn{2}{|c|}{$\begin{array}{c}100 \\
\text { (Human) }\end{array}$} \\
\hline & Ent & Rel & Ent & Rel & Ent & Rel & Ent & Rel & Ent & Rel & Ent & Rel & Ent & Rel & Ent & Rel \\
\hline Recall & 64 & 33 & 70 & 40 & 74 & 45 & 76 & 48 & 79 & 54 & 82 & 58 & 86 & 65 & 100 & 100 \\
\hline Pre. & 74 & 50 & 77 & 62 & 79 & 67 & 80 & 70 & 83 & 75 & 85 & 78 & 89 & 82 & 100 & 100 \\
\hline Value & 60 & 29 & 67 & 37 & 71 & 42 & 73 & 45 & 77 & 51 & 80 & 56 & 84 & 63 & 100 & 100 \\
\hline
\end{tabular}

Table 1: Precision, Recall and Value Scores for Entities and Relations for each DB Blend

\begin{tabular}{|l|c|c|r|r|r|r|r|c|}
\hline & $\begin{array}{c}\mathbf{0} \\
\text { (Machine) }\end{array}$ & $\mathbf{1 6 . 5}$ & $\mathbf{3 3}$ & $\mathbf{4 1}$ & $\mathbf{5 0}$ & $\mathbf{5 8}$ & $\mathbf{6 7}$ & $\begin{array}{c}\mathbf{1 0 0} \\
\text { (Human) }\end{array}$ \\
\hline Entities & 17,117 & 18,269 & 18,942 & 19,398 & 19,594 & 19,589 & 19,440 & 18,687 \\
\hline Relations & 6,684 & 6,675 & 6,905 & 7,091 & 7,435 & 7,808 & 8,406 & 11,032 \\
\hline Descriptions & 18,666 & 18,817 & 19,135 & 19,350 & 19,475 & 19,639 & 19,752 & 20,376 \\
\hline
\end{tabular}

Table 2: Entity, Relation and Description Counts for each DB Blend

To validate the interpolation algorithm and blending procedure, we applied NIST's 2004 ACE Scorer to the eight extraction databases. Polynomial approximations were fitted against both the entity and relation extraction curves. Entity performance was found to vary linearly with $\mathrm{DB}$ blend $\left(\mathrm{R}^{2}=.9853\right)$ and relation performance was found to vary with the square of DB blend $\left(\mathrm{R}^{2}=.9961\right)$. Table 1 shows the scores for each blend, and Table 2 shows the counts of entities, relationships, and descriptions.

\subsection{Question Answering Task}

Extraction effectiveness was measured by how well a person could answer questions given a database of facts, entities, and documents. Participants answered four sets of questions using four databases. They accessed the database using BBN's FactBrowser (Miller, 2001) and recorded their answers and source citations in a separate tool developed for this study, AnswerPad.

Each database represented a different database quality. In some databases, facts were missing, or incorrect facts were recorded. Consequently, answers were more accessible in some databases than in others, and participants had to vary their question answering strategy depending on the database.

Participants were given five minutes to answer each question. To ensure that they had actually located the answer rather than relied on world knowledge, they were required to provide source citations for every answer. The instruc- tions emphasized that the investigation was a test of the system, and not of their world knowledge or web search skills. Compliance with these instructions was high. Users resorted to knowledge-based proper noun searches only one percent of the time. In addition, keyword search was disabled to force participants to rely on the database features.

\subsection{Participants}

Study participants were recruited through local web lists and at local colleges and universities. Participants were restricted to college students and recent graduates with PC (not Mac) experience, without reading disabilities, for whom English was their native language. No other screening was necessary because the design called for each participant to serve as his or her own control, and because opportunities to use world knowledge in answering the questions were minimized through the interface and procedures.

During the first two months of the study 23 participants were used to help develop questions, participant criteria, and the overall test procedure. Then, experiments were conducted comparing the 0, 33, 67, and 100 database blends (Experiment 1, 20 subjects); the 0, 16.5, 33, and 50 database blends (Experiment 2, 20 subjects), and the 33, 41, 50, and 58 database blends (Experiment 3, 24 subjects). 


\subsection{Question Selection and Validation}

Questions were developed over two months of pilot studies. The goal was to find a set of questions that would be differentially supported by the $0,33,67$, and 100 databases. We explored both "random" and "engineered" approaches. The random approach called for creating questions using only the documents, without reference to the kind of information extracted. Using a list of keywords, one person generated 86 questions involving relationships and entities pertaining to politics and the military by scanning the 946 ACE documents to find references to each keyword and devising questions based on the information she found.

The alternative, engineered approach involved eliminating questions that were not supported by the types of information extracted by SERIF, and generating additional questions to fit the desired pattern of increasing support with increased human annotation. This approach ensured that the question sets reflected the structural differences that are assumed to exist in the database, and produced psychophysical data that link degree of QA support to human performance parameters. The IE results from four of the databases $(0,33,67$ and 100$)$ were used to develop questions that received differential support from the different quality databases. For example, such a question could be answered using the automatically extracted results, but might be more straightforwardly answered given human annotation.

Sixty-four questions, plus an additional ten practice questions, were created using the engineering approach. Additional criteria that were followed in creating the question sets were: 1) Questions had to contain at least one reasonable entry hook into all four databases, e.g., the terms U.S. and America were considered too broad to be reasonable; and, 2) For ease of scoring, listtype questions had to specify the number of answers required. Alternative criteria were considered but rejected because they correlated with the aforementioned set. The following are examples of engineered questions.

- Identify eight current or former U.S. State Department workers.

- In what two West Bank towns does Fatah have an office?
- Name two countries where Osama bin Laden has been.

- Were Lebanese women allowed to vote in municipal elections between two Shiite groups in the year 1998?

Two question lists, one with 86 questions generated by the random procedure and one with 64 questions generated by the engineered procedure, were analyzed with respect to the degree of support afforded by each of the four databases as viewed through FactBrowser. Four a priori criteria were established to assess degree of support - or its opposite, the degree of expected difficulty - for each question in each of the four databases. Ranked from easiest to hardest, they are listed in Table 3.

The question can be answered...

1. Directly with fact or description (answer is highlighted in FactBrowser citation)

2. Indirectly with fact or description (answer is not highlighted)

3. With name mentioned in question (long list of mentions without context)

4. Via database crawling

Table 3: A Priori Question Difficulty Characteristics, listed from easiest to hardest

Table 4 shows the question difficulty levels for both question types, for each of four databases. Analysis of the engineered set was done on all 64 questions. Analysis for randomly generated questions was done on a random sample of 44 of the 86 questions. Fifteen questions did not meet the question criteria, leaving 29 .

The randomly generated questions showed a statistically significant, but small, variation in expected difficulty, in part due to the number of unanswerable questions. While the questions were made up with respect to information found in the documents, the process did not consider the types of extracted entities and relations. This problem might have been mitigated by limiting the search to questions involving entities and relations that were part of the extraction task.

By contrast, the engineered question set showed a highly significant decrease in expected difficulty as the percentage of human annotation in the database increased $(\mathrm{P}<0.0001$ for chisquare analysis). This result is not surprising, given that the questions were constructed with reference to the list of entities in the four data- 
bases. The analysis confirms that the experimental manipulation of different degrees of support provided by the four databases was achieved for this question set.

\begin{tabular}{|c|c|c|c|c|}
\hline \multicolumn{5}{|c|}{ Random Question Generation } \\
\hline Difficulty & $0 \%$ & $33 \%$ & $67 \%$ & $100 \%$ \\
\hline $\begin{array}{l}\text { Level } \\
\text { (easiest to } \\
\text { hardest) }\end{array}$ & Human & Human & Human & Human \\
\hline 1 Fact- & 7 & 10 & 13 & 15 \\
\hline Highlight & & & & \\
\hline $\begin{array}{l}2 \text { Fact- } \\
\text { Indirect }\end{array}$ & 14 & 10 & 8 & 10 \\
\hline 3 Mention & 3 & 5 & 2 & 1 \\
\hline 4 Web Crawl & 5 & 4 & 6 & 3 \\
\hline Total & 29 & 29 & 29 & 29 \\
\hline \multicolumn{5}{|c|}{ Engineered Question Generation } \\
\hline Difficulty & $0 \%$ & $33 \%$ & 67 & $100 \%$ \\
\hline $\begin{array}{l}\text { Level } \\
\text { (from easiest } \\
\text { to hardest) }\end{array}$ & Human & Human & Human & Human \\
\hline $\begin{array}{l}1 \text { Fact- } \\
\text { Highlight }\end{array}$ & 16 & 25 & 35 & 49 \\
\hline $\begin{array}{l}2 \text { Fact- } \\
\text { Indirect }\end{array}$ & 23 & 20 & 18 & 14 \\
\hline 3 Mention & 7 & 14 & 11 & 1 \\
\hline 4 Web Crawl & 18 & 5 & 0 & 0 \\
\hline Total & 64 & 64 & 64 & 64 \\
\hline
\end{tabular}

Table 4: Anticipated Difficulty of Questions as a Function of Database Quality

Preliminary human testing with both question sets suggested that the $a$ priori difficulty indicators predict human question answering performance. Experiments with the randomly generated questions, therefore, were unlikely to reveal much about the databases or about human question answering performance. On the other hand, an examination of how different levels of database quality affect human performance, in a psychophysical experiment where structure is varied systematically, promised to address the question of how much support is needed for good performance.

Based on the question difficulties, and pilot study timing and performance results, the 64 questions were grouped into four, 16-question balanced sets.

\subsection{Procedure}

Participants were tested individually at our site, in sessions lasting roughly four hours. Training prior to the test lasted for approximately a half hour. Training consisted of a walk-through of the interface features followed by guided practice with sample questions. The test consisted of four question sets, each with a different database. Participants were informed that they would be using a different database for each question set and that some might be easier to use than others.

Questions were automatically presented and responses were captured in AnswerPad, a software tool designed for the study. AnswerPad is shown in Figure 2.

Key features of the tool include:

- Limiting view to current question set disallowing participants to view previous question sets

- Automatically connecting to correct db

- Logging time spent on each question

- Enforcing five-minute limit per question

- Enforcing requirement that all answers include a citation

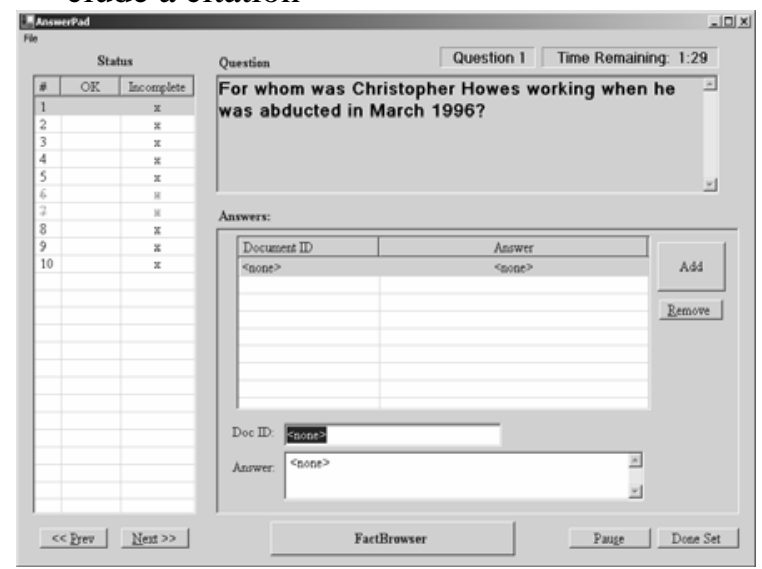

Figure 2: AnswerPad Question Presentation and Answer Capture Interface

Participants were given written documentation as part of their training. The participants were instructed to cut-and-paste question answers and document citations from source documents into AnswerPad.

Extracted facts and entities, and source documents were accessed through FactBrowser. FactBrowser, shown in Figure 3, is web-browser based and is invoked via a button in AnswerPad. FactBrowser allows one to enter a string, which 
is matched against the database of entity mentions. The list of entities that have at least one mention partially matching the string are returned (e.g., "Laura Bush”) along with an icon indicating the type of the entity and the number of documents in which the entity appears. Clicking on the entity in the left panel causes the top right panel to display all of the descriptions, facts, and mentions for the entity. Selecting one of these displays citations in which the description, fact, or mention occurs. Clicking on the citation opens up a document view in the lower right corner of the screen and highlights the extracted information in the text. When a document is displayed, all of the entities detected in the document are listed down the left side of the document viewer.

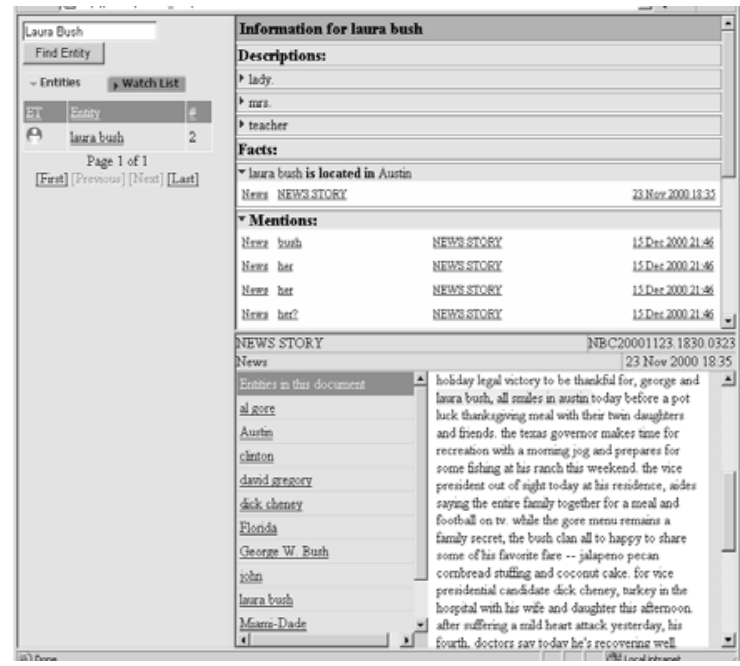

Figure 3: Browsing Tool Interface

The browsing tool was instrumented to record command invocations so that the path a participant took to answer a question could be recreated, and the participant's adherence to protocol could be verified. Furthermore, the find function (Ctrl-F) was disabled to prevent users from performing ad hoc searches of the documents instead of using the extracted data.

The order of question sets and the order of database conditions were counterbalanced across participants, so that, for every four participants, every question set and database appeared once in every ordinal position, and every question set was paired once with every database. This avoided carryover effects from question order.

\subsection{Data Collected}

Based on the initial results from Experiment 1, a $70 \%$ target effectiveness threshold was identified to occur between the 33 and 67 database blends. To refine and verify this finding, Experiment 2 examined the $0,16.5,33$, and 50 database blends. Experiment 3 examined the 33, 41, 50, and 58 database blends.

AnswerPad collected participant-provided answers to questions and the corresponding citations. In addition, AnswerPad recorded the time spent answering the questions. A limit of five minutes was imposed based on pilot study results. The browsing tool logged commands invoked while the user searched the fact-base for question answers. Questions were manually scored based on the answers in the provided corpus. No partial credit was given. The maximum score, for each database condition, was 16 , for a total maximum score of 64 .

\section{Results}

Figure 4 shows the question answer scores and times for each of the three individual experiments, and for Experiments 1 and 2 combined. Database quality affects both task speed (downward-sloping line) and task accuracy (upward-sloping line) in the expected direction. A logistic fit, as for a binary-response curve, was used to fit the relationship between blend percentage and accuracy in each experiment. The logistic fit Goodman-Theil quasi- ${ }^{2}$ was .9973 for Experiment 1, .9594 for Experiment 2, .8936 for Experiment 3, and .9959 for Experiments 1 and 2 combined.

For the target accuracy of $70 \%$, the $95 \%$ confidence interval for the required blend is $(35,56)$ around a predicted $46 \%$ blend for Experiment 1, and $(41,56)$ around a predicted $49 \%$ for Experiments 1 and 2 combined. 

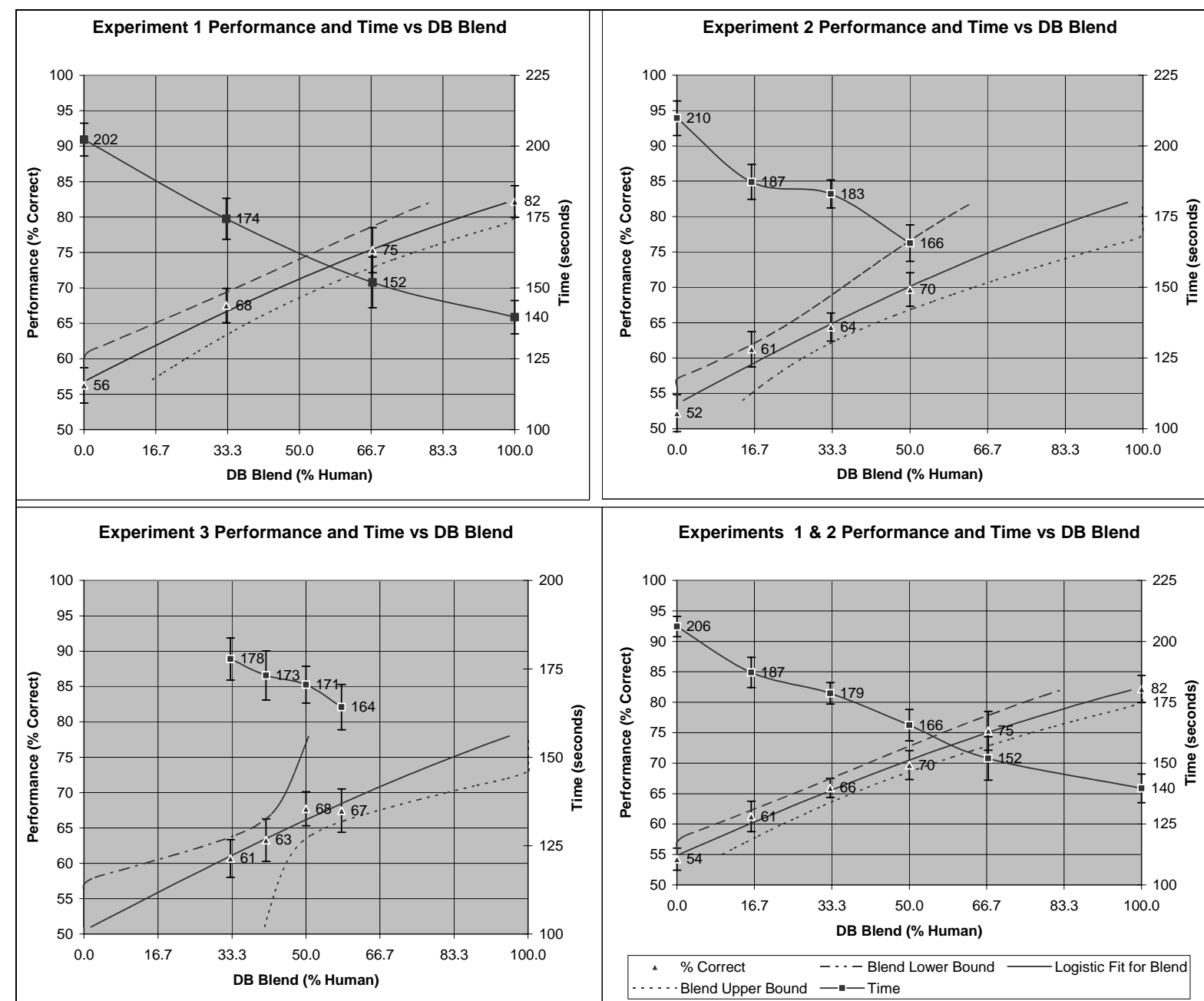

Experiments 1 \& 2 Performance and Time vs DB Blend

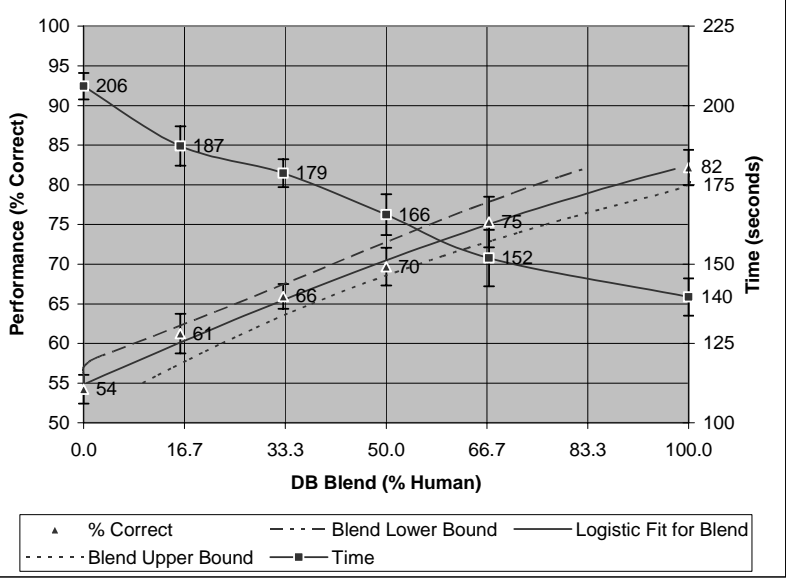

Figure 4 QA Performance (upward-sloping) and QA Time (downward-sloping) vs. Extraction Blend Error Bars are Plus/Minus Standard Error of Mean (SEM) Within Each Blend

Upper and Lower Bounds Are Approximate 95\% Confidence Intervals Based on the Logistic Fit For the Blend (X) to Produce a Given Performance (Y)

(Read these bounds horizontally, as bounds on $\mathrm{X}$, with the upper bound to the right of the lower bound.)

The downward-sloping line in each graph displays the average time to answer a question as a function of the extraction blend. For this analysis we used strict time, the time it took the participant to answer the question if he or she answered correctly, or the full 5 minutes allowed for any incorrectly answered question. This addresses the situation where a person quickly answers all of the questions incorrectly. The average question-answer time drops $32 \%$ as one moves from a machine generated extraction database to a human generated database. A straight-line fit to the Experiment 1 and 2 combined data predicts a drop of 6.5 seconds as the human proportion of the database increases by 10 percentage points.
A one-way repeated measures analysis of variance (ANOVA) was performed for Experiment 1 (0-33-67-100), Experiment 2 (0-16.5-3350), and Experiment 3 (33-41-50-58). Table 5 summarizes the results. In Experiments 1 and 2 the impact of database quality on QA performance and on QA time were highly significant ( $\mathrm{P}$ $<0.0001$ ), but not for the narrower range of databases in Experiment 3. Other ANOVAs showed that the impact of trial order and question set on QA performance were both nonsignificant $(\mathrm{P}>0.05)$. 


\begin{tabular}{lll}
\hline Experiment & $\begin{array}{l}\text { QA } \\
\text { Performance }\end{array}$ & Strict Time \\
\hline 1 & $\mathrm{~F}(3,57)=30.98$, & $\mathrm{F}(3,57)=28.36$ \\
& $\mathrm{P}<.0001$ & $\mathrm{P}<.0001$ \\
\hline 2 & $\mathrm{~F}(3,57)=19.32$, & $\mathrm{F}(3,57)=15.37$, \\
& $\mathrm{P}<.0001$ & $\mathrm{P}<.0001$ \\
\hline 3 & $\mathrm{~F}(3,69)=2.023$, & $\mathrm{F}(3,69)=1.053$, \\
& $\mathrm{P}=.1187$ & $\mathrm{P}=.3747$ \\
\hline
\end{tabular}

Table 5: ANOVA Analyses for QA Performance Expt. 1 used db blends of 0, 33, 67, and 100\%

Expt. 2 used db blends of 0, 16.5, 33, and 50\%

Expt. 3 used db blends of 33, 41, 50, and 58\%

In Experiment 1, Newman-Keuls contrasts indicate that the $0,33,67$, and 100 databases differ significantly $(\mathrm{P}<.05)$ on their impact on QA quality. For Experiment 2, however, the 16.5 and 33 database qualities were not shown to be different, nor were any of the database blends in Experiment 3. The data suggest that nearly half the improvement in QA quality from 0 to 100 occurs by the 33 database blend, and more than half the improvement in QA quality from 0 to 50 occurs by the 16.5 blend: a little "human" goes a long way. Experiment 3 suggests that small differences in data blends make no practical difference in the results. Alternatively, there might be real differences that are small enough such that a larger number of participants would be required to detect them. Experiment 3 also had two participants with atypical patterns of QA against blend, which might account for the failure to detect a difference between the 33 and 50 or 58 blends as suggested by the results from Experiment 2. Furthermore, larger experiments could reveal whether the atypical participants were representatives of a subpopulation, or simply outliers. Bearing the possibility of outliers in mind, we used the combination of Experiments 1 and 2 for the combined logistic analysis.

\section{Conclusions}

We presented a methodology for assessing information extraction effectiveness using an extrinsic study. In addition, we demonstrated how a novel database blending (merging) strategy allows interpolating extraction quality from automated performance up through human accuracy, thereby decreasing the resources required to conduct effectiveness evaluations.
Experiments showed QA accuracy and speed increased with higher IE performance, and that the database blend percentage was a good proxy for ACE value scores. We emphasize that the study was not to show that IE supports QA better than other technologies, rather to isolate utility gains due to IE performance improvements.

QA performance was plotted against humanmachine IE blend and, for example, 70\% QA performance was achieved with a database blend between $41 \%$ and $46 \%$ machine extraction. This corresponded to entity and relationship value scores of roughly 74 and 47 respectively.

The logistic dose-response model provided a good fit and allowed for computation of confidence bounds for the IE associated with a particular level of performance. The constraints imposed by AnswerPad and FactBrowser ensured that world knowledge was neutralized, and the repeated-measures design (using participants as their own controls across multiple levels of database quality) excluded inter-participant variability from experimental error, increasing the ability to detect differences with relatively small sample sizes.

\section{Acknowledgement}

This material is based upon work supported in part by the Department of the Interior under Contract No. NBCHC030014. Any opinions, findings and conclusions or recommendations expressed in this material are those of the authors and do not necessarily reflect the views of the Department of the Interior.

\section{References}

S. Miller, H. Fox, L. Ramshaw, and R. Weischedel, "A Novel Use of Statistical Parsing to Extract Information from Text", in Proceedings of 1st Meeting of the North American Chapter of the ACL, Seattle, WA., pp.226-233, 2000.

S. Miller, S. Bratus, L. Ramshaw, R. Weischedel, and A. Zamanian. "FactBrowser Demonstration", Human Language Technology Conference, San Diego, 2001.

D. Jones and E. Walton, "Measuring the Utility of Human Language Technology for Intelligence Analysis,” 2005 International Conference on Intelligence Applications, McLean, VA May, 2005. 